\title{
Clinical Application of the Computer Picture Archiving and Communicating System to Stereotactic Radiosurgery
}

\author{
Tooru Shiga, Hiroki Shirato, Yoichi Kikuchi, Takeshi Nishioka, Toshihiro Kitahara, Satoshi Terae, \\ Noriaki Nishiyama, Xu Bo, and Kazuo Miyasaka
}

\begin{abstract}
Stereotactic radiosurgery, a rapidly evolving technology, provides precise high-dose irradiation to brain lesions and tumors. A new method using digital subtraction angiographic images of a personal computerimage display terminal interfaced to our picture archiving and communication system (PACS) performs measurements and calculations of the coordinates of the location of the center of the lesion to be treated. The time required to calculate the coordinates was performed by three methods: hand calculation, digitizer method, and PACS method. These three coordinates were compared in 10 cases. The hand calculation method required -50 minutes, the digitizer method required $\sim 10$ minutes, and the PACS method $\sim 3$ minutes. The difference is statistically significant $(P<.001)$. The three methods were equally accurate. This type of PACS application using inexpensive personal computers interfaced more directly to a PACS is an emerging trend that facilitates wider access to images and to manipulating them for specialized applications.
\end{abstract}

Copyright 1996 by W.B. Saunders Company

KEY WORDS: personal computer, picture archiving and communication system (PACS), radiosurgery, radiology, computer.

QTEREOTACTIC radiosurgery is a precise high-dose irradiation technique, which is effective in treating small brain lesions such as arterio-venous malformations. Very precise lesion localization is possible using a stereotactic frame in a diagnostic work-up performed just before the irradiation for treatment planning. This study reports the use of a personal computer-image display terminal on the Hokkaido University picture archiving and communication system (HU-PACS) to directly access and display images on which a computer program permits measurements between designated points and then automatically calculates the location of the lesion.

\section{MATERIALS AND METHODS}

$P A C S$. The HU-PACS has been in daily clinical operation at the Hokkaido University Hospital in Sapporo, Japan, since 1989 and is the world's first full-scale PACS. It consists of host computers, image workstations, and image databases. ${ }^{1}$ The configuration of PACS at Hokkaido University Hospital (HU-PACS) is shown in Fig 1. Two computed tomography scanners (CT), a magnetic resonance imaging scanner (MR), and 12 Fuji computed radiography units (FCR) are connected to HU-PACS.

In 1994 a PACS subsystem using a personal computer image display terminal (PC-IDT) was developed to permit access to HU-PACS images along with greater flexibility in manipulating them in new ways. One such subsystem includes a digital subtraction angiography unit and a computerized tomographic scanner, a personal computer image display terminal, and a multimedia server which are interconnected by an Ethernet network using the Transmission Control Protocol/Internet Protocol. MR, CT, and FCR images are obtained through a gateway computer.

The PC-IDT is an PC 9821 (Nippon Electric Corp, Tokyo, Japan) with a 17-inch unit multisynchronous display, 14 mbytes of RAM memory and an 120 -mbyte hard disk. The central processing unit of the terminal is an Intel $486 \mathrm{DX}$ with a clock speed is $33 \mathrm{Mhz}$, and the operating system of the PC-IDT is Microsoft Windows 3.1 (Microsoft Corp, Redmond, WA).

Radiosurgery lesion localization. A new program written in the Visual Basic language operates on images on the described PC-IDT. This PACS method is compared to two earlier methods, the hand-calculation method and the digitizer method.

In all three methods the patient is placed in the Komai type frame with plates shown in Fig 2 for imaging for radiosurgery lesion localization and during treatment. The algorithm used in our institution for stereotactic localization of lesions is the same as the one reported from Siddon et al. ${ }^{2}$ Attached to the frame are plates on which fiducial markers are placed on the corners in a $6 \times 6 \mathrm{~cm}$ square on the anterior, posterior, left, and right faces surrounding the patient's head. Two localization images are required. Usually, $x$-ray beams pass through the patient from anterior to posterior (A-P) and from right to left (R-L). One projection includes the target and the eight anterior and posterior markers, whereas the second projection includes the target and the eight right and left markers.

The origin of three-dimensional (3D) coordinates is at the center of the frame. Having known the 3D coordinates of these fiducial markers, the target point is calculated as the intersection of the lines connecting the points of the target projected on the plates. Because of digitizing errors and difficulty in identifying a unique projected target point on the two films, the lines (A-P) and (R-L) rarely intersect exactly. Therefore, the target position is defined as the

From the Department of Radiology, Hokkaido University School of Medicine, Sapporo, Japan.

Address reprint requests to Hiroki Shirato, MD, Department of Radiology, Hokkaido University School of Medicine, Kita-

15, Nishi-7, Kita-ku, Sapporo, 060 Japan.

Copyright $\odot 1996$ by W.B. Saunders Company

0897-1889/96/0903-0004\$3.00/0 


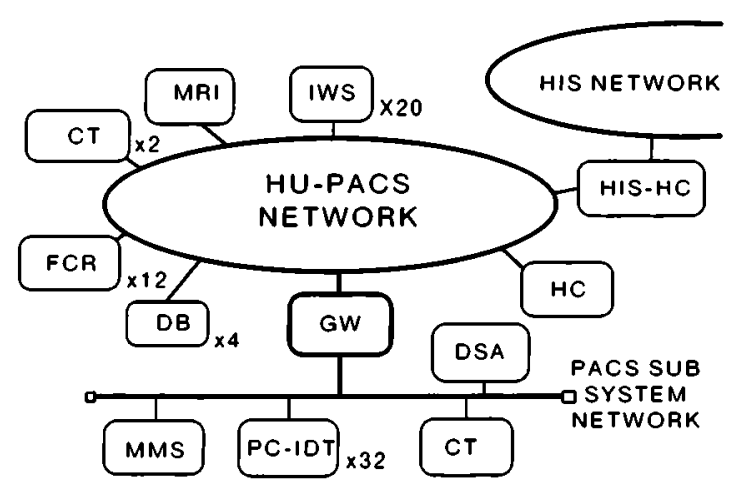

Fig 1. The configration of PACS at Hokkaido University Hospital. IWS, image workstation; HC, host computer; DB, data base; GW, gate way; MMS, multimedia server; HIS. hospital information system; FCR, Fuji Computed Radiogra. phy.

midpoint of the shortest line segment connecting the two lines.

Both of the older methods require radiographs be made and developed. In the hand-calculation method. used from 1991 to 1993. the coordinates were calculated by measuring the distance between the markers and the center of the lesion on the developed films. This method required considerable time and attention to detail.

In 1994. a program was developed that used a film digitizer and a mouse to allow the ctata to be input more spcedily. This digitizer method reduced both uncertainty and time required for calculation.

In our hospital. a CT scanner. a MRI scanner, digital subtraction angiography (DSA). and (R are located in the Division of Diagnostic Radiology on the first floor. whereas the linear accelerator and treatment planning room for radiosurgery are located in the basement (Fig 3). Because

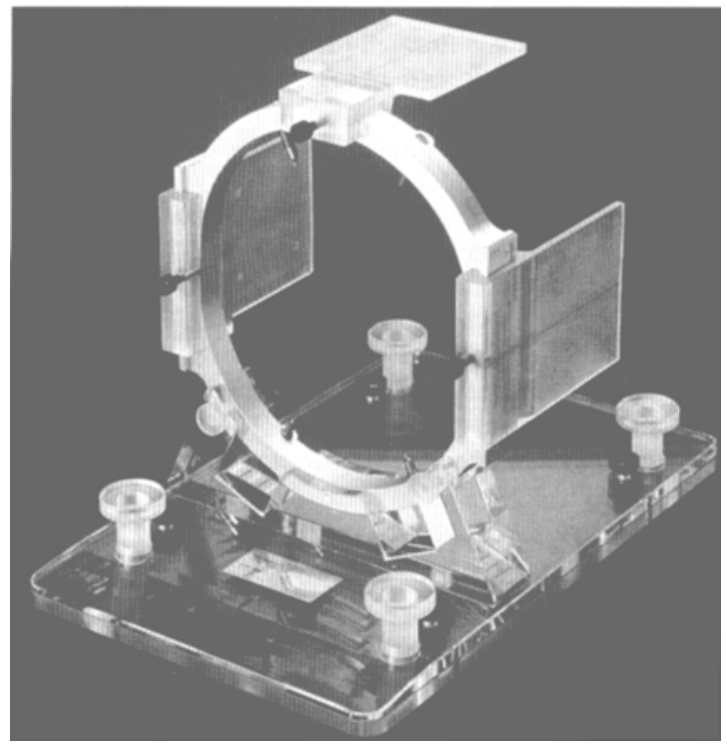

Fig 2. A komai type frame with plates for radiosurgery.

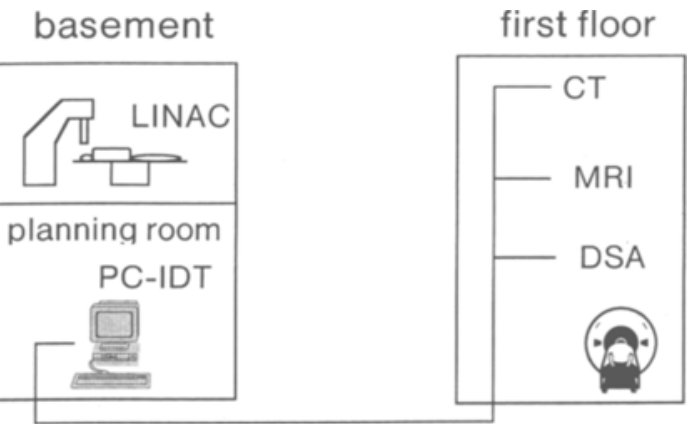

Fig 3. Location of equipment in the hospital.

radiosurgeons can now view CT. MRI, and DSA images on the PC.IDT in the planning room. using the PC.JDT in the planning room for localization made sense. The new program to determine the center of the lesion was developed in 1995. It was performed on $1.000 \times 1.000 \mathrm{DSA}$ images obtained directly from the HU.PACS.

A display showing the localization program is shown in Fig 4. After the selection of two images. click only the 8 marker points and the center of the lesion on each image with the mouse. Commands are easy for doctors to learn who are already familiar with MS-Windows and the PACS. subsystem. There are the usual utilities in the PACS subsystem. For example, the contrast and brightness of the image can be adjusted.

\section{RESULTS}

The time that elapsed from the end of the imaging to when the images bccame available for measurements was compared for the hand calculation and the digitizer method as opposed to the PACS method. In the hand calculation and the digitizer method, the film images must be developed before the coordinates can be calculated. In the PACS method, the images are sent through the Ethernet nctwork to the PCIDT in the treatment planning room. Neuroradiologists then determine the outer margin of the lesion at the console of the $1,000 \times 1,000$ DSA image on the terminal. The clapsed time was about 13 minutes for hand-calculation and the digitizer methods, whereas it took only about 7 minutes using the PACS method.

The time required for the measurements and the final determination of the coordinates was measured using each method in 10 patients. The means and the standard errors obtained are shown in Table 1. As can be seen, the PACS method shortens the time substantially, to about one tenth of the time required by hand calculation and to half of the time required by the digitizer method. The difference is statistically 
Fig 4. A display showing the localization program.

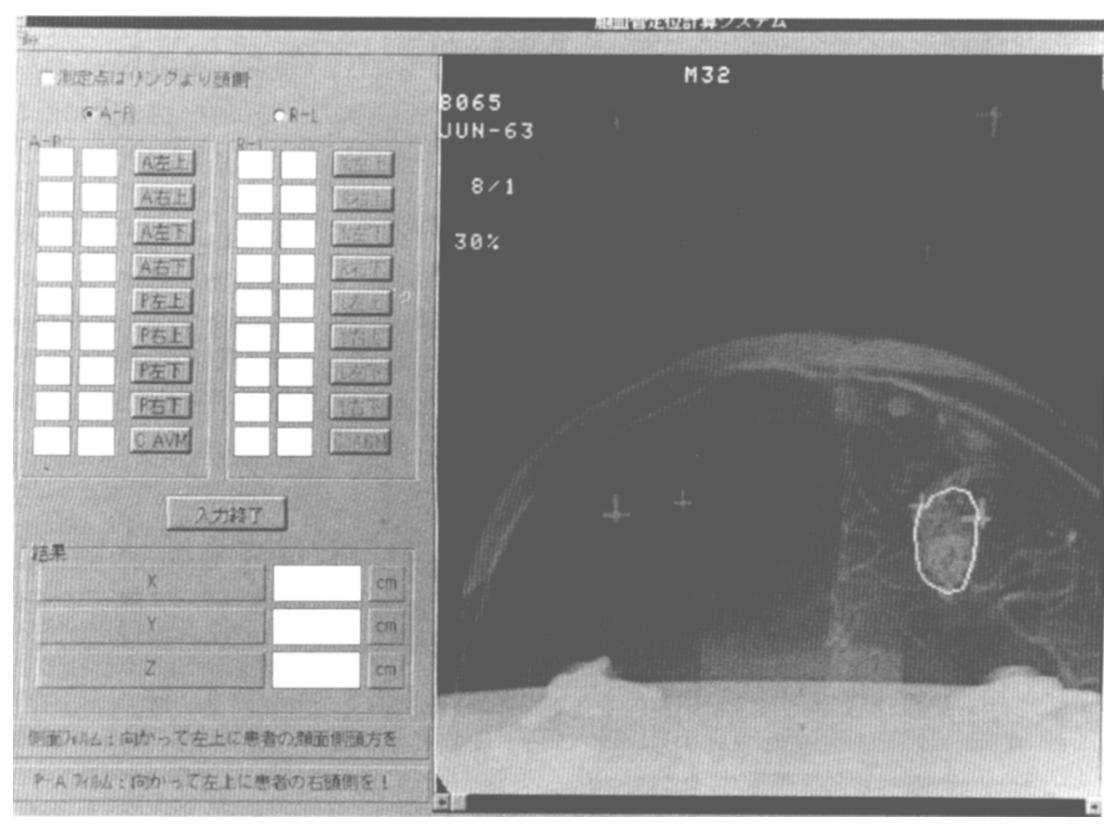

significant $(P<.001)$ between hand calculation and the digitizer method and between the digitizer method and the PACS method $(P<.001)$.

The calculated coordinates of the three methods were identical within plus or minus $1 \mathrm{~mm}$.

\section{DISCUSSION}

The new PACS-based method was superior to both the hand calculation and the digitizer methods in three ways. There were time savings from the end of imaging to the beginning of the measurements, and there were further time savings in the measurement and calculation operations. There was greater certainty in identifying the lesion and the markers, and the location of the PC-IDT in the radiosurgery planning room was a further convenience. The rapidity of the PACS method also facilitated redoing the measurements for confirmation of the coordinates.

Table 1. Time Required for the Measurement and the Final Determination of the Coordinates

\begin{tabular}{lcr}
\hline & Mean Time (min) & \multicolumn{1}{c}{1 SD } \\
\hline Hand calculation & 50.311 & 19.347 \\
Digitizer method & 11.524 & 1.207 \\
PACS method & 2.742 & 0.851 \\
\hline
\end{tabular}

Mean time and standard error of the time from the start of the calculation to the final determination of the coordinates using each method.
Our use of a personal computer workstation is part of an emerging trend. Although PACS vendors have offered lower end workstations built on personal computer platforms, the use of personal computers directly with minimal interfacing means further significant cost reduction. These lower costs made it possible to put a PC-IDT in every ward of the hospital. In applications where somewhat lower image resolution is satisfactory, this approach is likely to be more widely used to provide access to images both inside and outside of Radiology. MR Ramaswarmy ${ }^{3}$ has reported that the personalcomputer PACS gives easy access to image information, so activities such as image analysis, data processing, and maintaining personal research files can be done easily by a personal computer.

Many of the installed PACS, including HUPACS, have little flexibility in programming new functions and in having wide access to images because of the high cost of the usual workstations offered by vendors. The trend toward personal computer use permits not only wider access to images, but also to the raw image data sets. As programming on such images in the PC-IDT does not conflict with the complex central programs, substantial new opportunities for specialized applications exist. ${ }^{4}$ It was notable that the time required to develop 
this specific program was only 3 weeks. Such developments will enhance existing PACS by helping to move from largely one-way information display system to a communicating system more useful for patient care and research. ${ }^{5}$

The PC-IDT used in this study was limited in speed, memory size, and ability to display more than one image at a time. These were not significant problems for this application. The current PC-IDT will soon be replaced by Pentium-based computers (Intel Corp, USA) so that these disadvantages will be decreased.
These units cost only one-thirtieth that of the workstations sold for full interpretation. Next we are planning to incorporate CT and MRI images into the lesion localization routines. ${ }^{6}$

In conclusion, PACS subsystem supplements to HU-PACS have been shown to be useful in radiosurgery planning, one of the rapidly developing fields of modern clinical medicine. Personal computers used on PACS have the versatility to facilitate many specialized medical uses by end users.

\section{REFERENCES}

1. Irie G, Miyasaka K: Clinical Experience-16 months of HU-PACS. Comp Med Imaging and Graphics 15:191195,1991

2. Siddon RL, Norman H, Barth NH: Stereotaxic localization of intracranial targets. Int J Radiat Oncol Biol Phys 13:1241-1246, 1987

3. Ramaswamy MR, Wong AWK, Lee JK, et al: Accessing picture archiving and communication system text and image information through personal computers. Am J Roentgenol 163:1239-1243, 1994
4. Frank MS, Berge R, Stern EJ, et al: Integrating a Personal-Computer Local-Area Network with a Radiology Information System: Value as a Tool for Clinical Research. Am J Roentgenol 162:709-712, 1994

5. Irie G: Concept of Hokkaldo University picture archiving communication system. J Digit Imaging 4:3-5, 1991

6. Shirato H, Suzuki K, Nishiok T, et al: Precise positioning of intracranial small tumors to the linear accelerator's isocenter, using a stereotactic radiotherapy computed tomography system (SRT-CT). Radiat Oncol 32:180-183, 1994 